\title{
ON PASTING BALLS TO HANDLEBODIES
}

BY C. D. FEUSTEL

Communicated by Franklin P. Peterson, November 17, 1969

Throughout this paper all spaces will be simplicial complexes and all maps will be piecewise linear. We shall denote the boundary, closure, and interior of a space $X$ by $b d(X), \operatorname{cl}(X)$ and $\operatorname{int}(X)$ respectively. Let $X$ be a space and $Y$ a connected subspace. Then we shall denote the natural map induced by inclusion from $\pi_{1}(Y)$ into $\pi_{1}(X)$ by $\pi_{1}(Y) \rightarrow \pi_{1}(X)$.

We shall say that a submanifold $X$ of a manifold $Y$ is properly embedded in $Y$ if $X \cap \operatorname{bd}(Y)=\mathrm{bd}(X)$. A handlebody is a 3-manifold homeomorphic to the regular neighborhood of a compact 1-complex embedded in $E^{3}$. If $T_{n}$ is a handlebody and $l$ is a simple loop in $\operatorname{bd}\left(T_{n}\right)$, we can attach a disk to $T_{n}$ by identifying the boundary of the disk with $l$. We may attach a thickened disk or a ball in a similar way to $T_{n}$ and obtain a 3-manifold. When we perform the operation above we shall say that we have pasted a ball to $T_{n}$ along $l$. We shall denote the smallest normal subgroup of $\pi_{1}\left(T_{n}\right)$ containing $[l]$ by $N(l)$.

It is the purpose of this article to prove:

THEOREM. Let $T_{n}$ be a handlebody of genus $n$. Let $l$ be a simple loop in $\operatorname{bd}\left(T_{n}\right)$ such that $\pi_{1}\left(T_{n}\right) / N(l)$ is free on $n-1$ generators. Then the 3-manifold obtained by pasting a ball to $T_{n}$ along $l$ is a handlebody of genus $n-1$.

The author would like to thank C. D. Papakyriakopoulos for suggesting that the theorem above be stated so that its relationship to the classical Poincare Conjecture would be clear. The author would also like to thank P. Stebe for locating the algebraic result due to J. H. C. Whitehead which is cited below.

Proof. It follows from a theorem of Whitehead (see [2, p. 167, Theorem N3]) that [l] can be taken to be a generator of $\pi_{1}\left(T_{n}\right)$. Let $T_{n}^{\prime}$ be homeomorphic to $T_{n}$ under a map $h: T_{n} \rightarrow T_{n}^{\prime}$. Then we can paste $T_{n}$ to $T_{n}^{\prime}$ along regular neighborhoods in $\operatorname{bd}\left(T_{n}\right), \operatorname{bd}\left(T_{n}^{\prime}\right)$ of $l$ and $h(l)$ respectively, to obtain a 3-manifold $M$.

It is a consequence of Van Kampen's Theorem that $\pi_{1}(M)$ is free on $2 n-1$ generators. Now $\pi_{1}(\mathrm{bd}(M))$ is not free, so $\pi_{1}(\mathrm{bd}(M))$ $\rightarrow \pi_{1}(M)$ is not one-one. It follows from the loop theorem [3] that there is a disk properly embedded in $M$ such that $\mathrm{bd}(D)$ is not

AMS Subject Classifications. Primary 5701, 5560.

Key Words and Phrases. 3-manifold, Poincaré conjecture, handlebody. 
homotopic to a point in $\mathrm{bd}(M)$. We pick $\mathbb{D}$ so that $\mathrm{bd}(\mathbb{D}) \cap \mathrm{bd}\left(T_{n}\right)$ $\cap \mathrm{bd}\left(T_{n}^{\prime}\right)$ is a finite set of minimal cardinality. (In particular, bd(D) crosses bd $\left(T_{n}\right) \cap \mathrm{bd}\left(T_{n}^{\prime}\right)$ at every point in the above set.) By general position $\operatorname{cl}\left(D \cap\left(\operatorname{bd}\left(T_{n}\right) \cap \operatorname{int}(M)\right)\right)$ may be taken to be a family of simple $\operatorname{arcs} \alpha_{1}, \cdots, \alpha_{m}$ whose interiors are pairwise disjoint. We may assume further that each of the above arcs meets $l$ in at most one point and that said arc crosses $l$ at this point. We may choose a component of $D-\bigcup_{i=1}^{m} \alpha_{i}$ whose closure contains at most one of the $\alpha_{i}$. The closure of this component is a disk $D_{1}$ which we may assume is properly embedded in $T_{n}$.

We claim that we may assume bd( $\left.\mathscr{D}_{1}\right)$ is not nullhomotopic in $\mathrm{bd}\left(T_{n}\right)$. This can be seen as follows: If $\mathrm{bd}\left(D_{1}\right)$ is nullhomotopic on bd $\left(T_{n}\right)$, it bounds a subdisk $D_{2}$ of bd $\left(T_{n}\right)$. Since $l$ is not nullhomotopic on bd $\left(T_{n}\right)$ and since $l$ meets bd $\left(D_{2}\right)$ at most once, bd( $\left.D_{2}\right)$ does not meet $l$. It follows that both endpoints of the $\operatorname{arc} b d\left(D_{2}\right) \cap b d(M)$ lie on a single loop $l_{1}$ of the boundary of a regular neighborhood of $l$ in $\operatorname{bd}\left(T_{n}\right)$. Now $l_{1} \cap D_{2}$ is an arc which separates $D_{2}$ into two disks. One of these disks lies on $\mathrm{bd}(M)$. It is now easily seen that $\mathrm{bd}(D)$ did not meet $\operatorname{bd}\left(T_{n}\right) \cap \mathrm{bd}\left(T_{n}^{\prime}\right)$ in a set of minimal cardinality. The claim follows.

Thus bd $\left(D_{1}\right)$ may be assumed to be nonnullhomotopic on $\operatorname{bd}\left(T_{n}\right)$. It follows from our assumptions above that $b d\left(D_{1}\right) \cap l$ is at most a single point. We will show that this intersection may be taken to be a single point.

Assume $D_{1}$ does not meet $l$, i.e., $D_{1}=D_{\text {. }}$

We remove a regular neighborhood of $D_{1} \cup h\left(D_{1}\right)$ from $M$. Denote the closure of the resulting manifold by $M_{1}$. Now bd $\left(M_{1}\right)$ is a closed surface in $M$. Since $\pi_{1}(M)$ is free, $\pi_{1}\left(\mathrm{bd}\left(M_{1}\right)\right) \rightarrow \pi_{1}(M)$ is not an injection whenever genus $\mathrm{bd}\left(M_{1}\right)>0$. Let $\lambda$ be a loop on $\mathrm{bd}\left(M_{1}\right)$ which is nullhomotopic in $M$. Now $M_{1} \cap \mathrm{cl}\left(M-M_{1}\right)$ is a collection of disks. It follows from Lemma 1.2 in [1] that $\lambda$ is nullhomotopic in $M_{1}$. It follows from the loop theorem in [3] that there exists a disk $E$ properly embedded in $M_{1}$ such that bd(E) is not nullhomotopic in $\mathrm{bd}\left(M_{1}\right)$. Clearly we may choose $\mathrm{bd}(E)$ to lie on $\operatorname{bd}\left(M_{1}\right) \cap \mathrm{bd}(M)$ since $\mathrm{bd}\left(M_{1}\right)-\mathrm{bd}(M)$ is a collection of disks. By the arguments above either we can find a sequence of compact 3-submanifolds $M_{i}$, $i=1, \cdots, m$, such that

(1) $M_{i} \supset M_{i}+1$ for $i=1, \cdots, m-1$;

(2) $l \subset \operatorname{bd}\left(M_{i}\right)$ for $i=1, \cdots, m$;

(3) genus $\operatorname{bd}\left(M_{i}\right)>$ genus $\operatorname{bd}\left(M_{i+1}\right)$ for $i=1, \cdots, m-1$;

(4) $\operatorname{bd}\left(M_{i+1}\right)-\operatorname{bd}\left(M_{i}\right)$ is the union of four open disks, for $i=1, \cdots, m-1$, and $m=\operatorname{genus} \operatorname{bd}(M) / 2$; or there is a disk $\bar{D}$ 
properly embedded in $T_{n}$ such that

(1) $\operatorname{bd}(\bar{D})$ is not nullhomotopic in $\mathrm{bd}\left(T_{n}\right)$;

(2) bd(D) $\cap l$ is a single point at which bd(D) crosses $l$.

The former is impossible since the genus of $\mathrm{bd}(M)$ is finite and since $l$ must be nonnullhomotopic on $\operatorname{bd}\left(M_{i}\right)$ for each $i$ by 1.2 in [1].

It follows that we can find a disk $D$ properly embedded in $T_{n}$ such that bd(D) is not nullhomotopic in $\mathrm{bd}\left(T_{n}\right)$ and $D \cap l$ is a single point at which bd(D) crosses $l$.

Let $H$ be a regular neighborhood of $D \cup l$. Now $\operatorname{cl}\left(\operatorname{bd}(H) \cap \operatorname{int}\left(T_{n}\right)\right)$ is a disk $E$ separating $H$ from $\mathrm{cl}\left(T_{n}-H\right)$. By Van Kampen's Theorem

$$
\begin{aligned}
\pi_{1}\left(T_{n}\right) & =\pi_{1}(H)_{\pi_{1}(E)}^{*} \pi_{1}\left(\mathrm{cl}\left(T_{n}-H\right)\right) \\
\prod_{i=1}^{n} Z & =Z * G
\end{aligned}
$$

where $G=\pi_{1}\left(\operatorname{cl}\left(T_{n}-H\right)\right)$. It follows that $G=\prod_{i=1}^{n-1} Z$.

Using the method of proof above one can find a sequence $B_{1}, \cdots$, $B_{m}$ of 3-submanifolds of $\operatorname{cl}\left(T_{n}-H\right)$ such that

(1) $B_{i} \supset B_{i+1}$;

(2) $\mathrm{bd}\left(B_{i+1}\right)-\mathrm{bd}\left(B_{i}\right)$ is the union of two open disks;

(3) $\mathrm{bd}\left(B_{i}\right) \supset E$;

(4) $\operatorname{cl}\left(\mathrm{cl}\left(T_{n}-H\right)-B_{i}\right)$ is a collection of $i$ 3-balls for all $i$;

(5) the total genus of $\operatorname{bd}\left(B_{m}\right)$ is zero. It follows that $\operatorname{cl}\left(T_{n}-H\right)$ is a handlebody of genus $n-1$.

The desired result now follows by choosing an appropriate embedding of $T_{n}$ in $E^{3}$ and pasting a ball to $T_{n}$ across $l$ in $E^{3}$.

\section{BIBLIOGRAPHY}

1. C. D. Feustel, Some applications of Waldhausen's results on irreducible surfaces, Trans. Amer. Math. Soc. 149 (1970), 575-583.

2. W. Magnus, A. Karrass and D. Solitar, Combinatorial group theory; Presentations of groups in terms of generators and relations, Pure and Appl. Math., vol. 13, Interscience, New York, 1966. MR 34 \#7617.

3. J. Stallings, On the loop theorem, Ann. of Math. (2) 72 (1960), 12-19, MR 22 \#12526.

Institute for Defense Analyses, Princeton, New Jersey 08541 